\title{
PACIENTE COM ACIDENTE VASCULAR CEREBRAL ISQUÊMICO JÁ É ATENDIDO COM MAIS RAPIDEZ NO HOSPITAL SÃO PAULO
}

\author{
Lilia Fumie Yamashita', Marcia Maiumi Fukujima², \\ Nina Granitoff', Gilmar Fernandes do Prado²
}

\begin{abstract}
RESUMO - Foram caracterizados os pacientes com diagnóstico de acidente vascular cerebral (AVC) isquêmico internados por período superior a 24 horas. Investigamos os fatores que influenciam a chegada precoce ou tardia do paciente ao pronto socorro, o intervalo de tempo entre a chegada e a realização de tomografia computadorizada (TC), os fatores que influenciam na permanência destes pacientes no hospital e o seu destino. Concluímos que a população atendida pelo Hospital São Paulo tem chegado ao hospital mais precocemente que há 3 anos ( $47 \%$ chegaram nas primeiras 3 horas de instalação) e que o fluxo interno do paciente ficou muito mais rápido, uma vez que o AVC é considerado emergência médica pela equipe de saúde. As principais complicações apresentadas pelos pacientes foram infecciosas e nos direcionam à proposta de implementação de protocolos para a melhoria aos cuidados que devem ser prestados pelos profissionais de saúde.
\end{abstract}

PALAVRAS-CHAVE: acidente vascular cerebral, isquemia cerebral, complicações.

\section{Patients with ischemic stroke are taken care quickly in São Paulo Hospital}

ABSTRACT - We observed inpatients with the diagnosis of ischemic stroke hospitalized at least 24 hours. We investigated the reasons for early or late arrival to the Emergency Room, the time span from arrival to computed tomography (CT) scanning, factors associated to hospitalization time, and patients outcome. We concluded that people who are cared for by São Paulo Hospital now get to the hospital sooner than they did 3 years ago ( $47 \%$ arrived in 3 hours or less) and that the patient flow inside the hospital became much faster, once any stroke patient is considered now a medical emergency by the hospital staff. Infections were the main complications presented by our patients, which is leading us to optimize protocols to improve emergency care by all the staff.

KEY WORDS: stroke, brain ischemia, complications.

Doença vascular encefálica é a primeira causa de incapacidade e terceira causa de mortalidade nos Estados Unidos, com aproximadamente 600000 casos e 160000 mortes ocorrendo anualmente, precedido apenas por doenças cardíacas e câncer ${ }^{1}$. As doenças cerebrovasculares persistem como a primeira causa de morte na Escócia, na Itália, no Japão, em países do leste europeu e na China². A incidência no mundo foi estimada em 300 casos a cada 100000 pessoas, com discreta predominância para os homens, para população estudada entre 1980 a 1990 e com idade entre 45 e 84 anos $^{3}$. De acordo com estudo realizado no Brasil entre 1980 a 1995, as doenças cerebrovasculares correspondem a um terço dos óbitos anuais por doen- ças do aparelho circulatório ${ }^{3}$. O custo anual do tratamento agudo e crônico dos pacientes que sofrem acidente vascular cerebral (AVC) é de aproximadamente trinta bilhões de dólares somente nos Estados Unidos, sendo dezessete bilhões com custos médicos e treze milhões com custos indiretos $^{4}$. Entre 1994 e 1997, o número absoluto de hospitalizações por AVC no Brasil variou entre 198705 e 295596 por ano, estimando-se que $25 \%$ destes tenham sido doenças cerebrovasculares recorrentes. A tendência das hospitalizações, crescente, com isso, os custos dos pacientes com AVC aumentaram em 9,7\% entre 1995 e 1997 no país 3 .

As estratégias terapêuticas do AVC resumemse ao cuidado agudo que visa limitar a progres-

Setor de Urgência do Hospital São Paulo / Escola Paulista de Medicina / Universidade Federal de São Paulo (UNIFESP): ${ }^{1}$ Enfermeira, Departamento de Enfermagem; ${ }^{2}$ Neurologista, Disciplina de Neurologia.

Recebido 7 Abril 2003, recebido na forma final 8 Agosto 2003. Aceito 20 Setembro 2003.

Dra. Lilia Fumie Yamashita - Rua Napoleão de Barros 754 - 04024-002 São Paulo SP - Brasil. 
são da lesão, promover neuroproteção ${ }^{6}$ e prevenir a recorrência ${ }^{5}$. Até a década de 80 , os cuidados limitavam-se às medidas de suporte clínico e à reabilitação.

Recentes evidências experimentais e clínicas sugerem que a persistência da isquemia cerebral por mais de 4 a 6 horas produz lesões neurológicas permanentes ${ }^{7}$. Com a aprovação da terapia trombolítica por meio do fator ativador tecidual de plasminogênio (rt-PA) para pacientes com $A V C$ isquêmico $(A V C l)$ realizado nas primeiras 3 horas de início dos sinais e sintomas ${ }^{8}$, diversos estudos têm sido desenvolvidos para caracterizar esta população. Os objetivos do atendimento inicial no doente com AVC agudo são: a confirmação do diagnóstico, a identificação da hora de início do quadro e da sua evolução e a graduação da gravidade do acidente vascular cerebral ${ }^{9}$. A avaliação na urgência inclui ainda a estabilização das condições vitais do paciente, tais como cuidados respiratórios, balanço hidroeletrolítico, monitorização hemodinâmica, codições dietéticas, controle rigoroso da temperatura e da glicemia e prevenção de trombose venosa profunda ${ }^{11}$. Em estudo realizado no Hospital São Paulo (HSP) no período de 1998 a 1999, antes da utilização de trombolítico, verificou-se o grande intervalo de tempo no atendimento a pacientes com diagnóstico de AVC, mostrando que a doença não era considerada emergência médica ${ }^{10}$.

Este estudo visa caracterizar os pacientes vítimas de $\mathrm{AVCl}$ agudo atendidos num hospital universitário terciário quanto ao tempo decorrido até $o$ atendimento, o fluxo interno e as principais complicações.

\section{MÉTODO}

Analisamos dados clínicos, epidemiológicos, demográficos intra-hospitalares e sociais considerados relevantes para o atendimento do paciente com AVC na realidade urbana de uma grande metrópole.

Local: Pronto Socorro do HSP, hospital de nível terciário, considerado oficialmente como referência para o atendimento de urgência/emergência no município de São Paulo.

Período: maio a agosto de 2002.

Critério de inclusão: estar internado no pronto socorro por mais de 24 horas com o diagnóstico de $\mathrm{AVCI}$.

Critérios de exclusão: transferência de outros Estados ou outros hospitais; outros diagnósticos neurológicos diferentes de AVCl.

Desenho de estudo: estudo transversal com inclusão consecutiva de casos.

Coleta de dados: obteve-se através de entrevista direta dados de identificação (idade, sexo, cor), fatores de risco (hipertensão arterial, diabetes mellitus, tabagismo, dislipidemia, AVC prévio, ataque isquêmico transitório, doenças cardíacas, sem fatores de risco e outros), quadro clínico, meios de transporte utilizados (transporte privado, público ou ambulância) e as principais dificuldades encontradas até a chegada ao hospital (desconhecimento da gravidade, falta de transporte, viver/morar sozinho, congestionamento e outros). Os dados relacionados às complicações clínicas (broncoaspiração, pneumonia, infecção do trato urinário, hipotensão, hipertensão, hipertermia, arritmia, hipoglicemia, hiperglicemia, rebaixamento do nível de consciência, transformação hemorrágica e outros), aos procedimentos invasivos a que foram submetidos (via aérea definitiva, sondagem vesical, sondagem gástrica e acesso venoso central) e ao intervalo de tempo entre a chegada ao hospital e realização da tomografia computadorizada (TC) foram obtidos através de análise do prontuário. O prontuário do HSP registra a hora de chegada na sua ficha de admissão e o departamento de diagnóstico por imagem registra a hora de revelação do filme da TC em um de seus equipamentos. Quando não realizado em equipamento com este dispositivo, o intervalo para TC foi obtido, quando possível, através do prontuário. O paciente foi acompanhado durante todo o período de sua permanência hospitalar.

Análise estatística: Para verificar se as distribuições são de tipo normal, foi aplicado o teste de aderência de Kolmogorov-Smirnov. Para verificar a diferença entre os tempos de realização da TC no grupo de pacientes que chegaram ao serviço em até 3 horas e mais que 3 horas foi usado teste de Mann-Whitney. Consideramos como significante $p<0,05$.

\section{RESULTADOS}

Trinta e oito pacientes com diagnóstico de AVC isquêmico foram incluídos consecutivamente neste estudo, conforme os critérios descritos. Vinte e dois pacientes $(57,9 \%)$ eram do gênero feminino e $16(42,1 \%)$ do masculino. Deste total, 25 $(65,8 \%)$ eram de cor branca, $3(7,9 \%)$ de cor negra e $10(26,3 \%)$ de cor parda. Não houve pacientes de cor amarela.

Foram analisados os principais fatores de risco já conhecidos no momento do atendimento. Dos 38 pacientes, $31(81,6 \%)$ apresentavam hipertensão arterial, $22(57,9 \%)$ alguma doença cardíaca, $14(36,8 \%)$ já sofreram AVC prévio, 13 (34,2\%) eram ou já foram tabagistas, $10(26,3 \%)$ apresentavam diabetes mellitus, 3 (7,9\%) eram dislipidêmicos, um $(2,6 \%)$ sofreu ataque isquêmico transitório (AIT) prévio, $2(5,3 \%)$ não apresentavam fatores de risco e $6(15,8 \%)$ apresentavam outros 


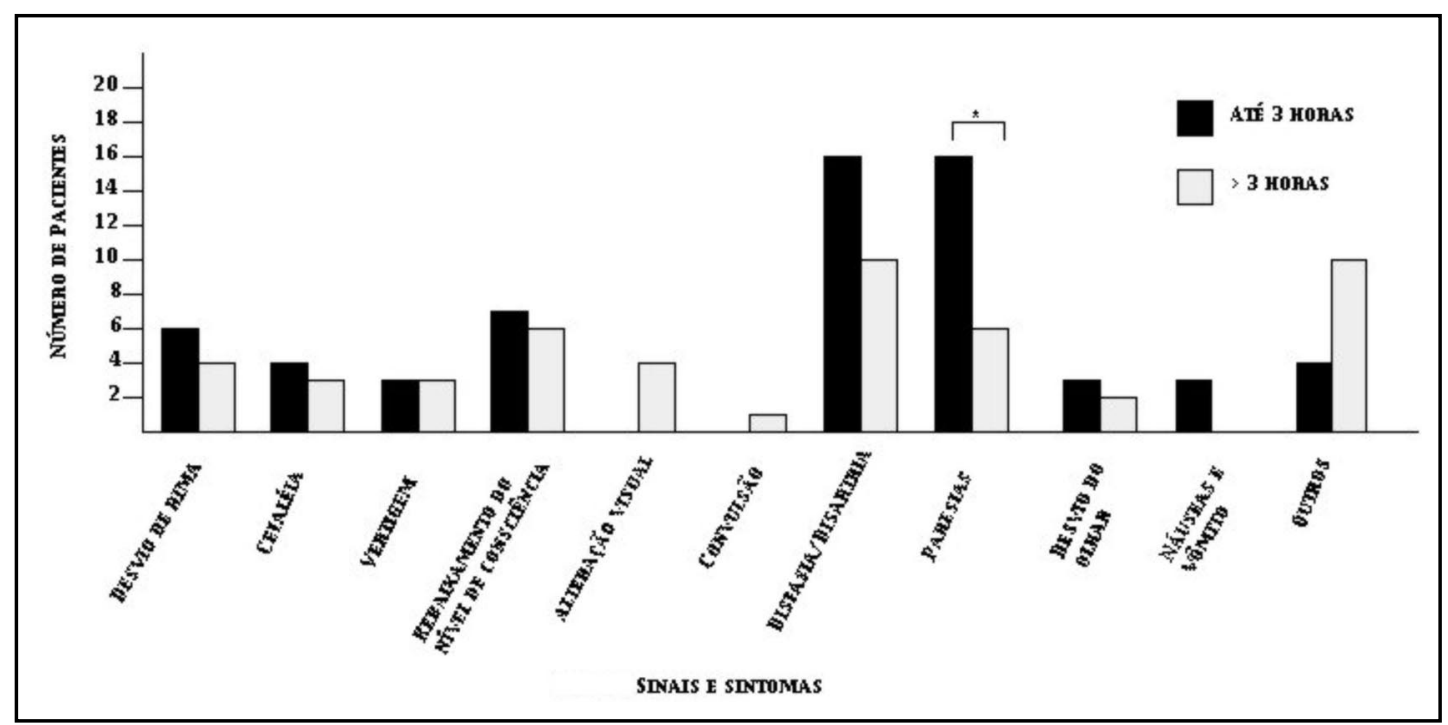

Fig 1. Número de paciente relacionado com os sinais e sintomas apresentados e o intervalo entre o ínicio dos sinais e sintomas e chegada ao hospital. Teste de Kolmogorov-Smimov $(* p<0,05)$

fatores, tais como: história familiar de AVC, etilismo, obesidade e lúpus eritematoso sistêmico.

A média do intervalo de tempo entre o início dos sintomas e a chegada ao hospital foi de 10 horas e 7 minutos. Dezoito pacientes $(47,4 \%)$ procuraram o serviço médico nas primeiras 3 horas de início dos sinais e sintomas, $4(10,5 \%)$ chegaram ao hospital entre 3 e 6 horas, $11(28,9 \%)$ chegaram entre 6 e 24 horas e $4(10,5 \%)$ apresentaram-se apenas 24 horas após o início de sinais e sintomas. Não foi possível obter esta informação em um paciente $(2,6 \%)$.

O meio de transporte mais utilizado foi o particular, através de veículo próprio (familiar ou conhecido), correspondendo a 30 casos $(78,9 \%)$. Cinco pacientes $(13,2 \%)$ foram trazidos pelo sistema público de ambulância, $2(5,3 \%)$ utilizaram transporte público (ônibus ou metrô). Não foi possível obter esta informação de um $(2,6 \%)$ paciente.

Os principais sinais e sintomas que levaram o paciente a procurar o serviço médico, relacionado ao tempo de chegada ao hospital, estão ilustrados na Figura 1. O teste de KolmogorovSmirnov mostra diferença no tempo de chegada para os indivíduos que apresentam paresias $(p<$ $0,05)$. Na categoria outros encontramos: perda da coordenação, tremor, dispnéia, mal estar epigástrico, otalgia, agitação psico-motora, liberação esfincteriana, parestesia, "enrolar a língua", hipotensão, versão da cabeça, disfagia e ptose palpebral. Observou-se que $56,4 \%$ dos sinais e sintomas ocorreram em pacientes que se apresenta-
Tabela 1. Relação entre o intervalo de tempo entre o início dos sintomas até à chegada ao serviço de emergência e o tempo para a realização da tomografia computadorizada (TC) desde a chegada ao hospital.

Tempo de TC ( $\min )$

\begin{tabular}{ccc} 
Chegada ao HSP & $\begin{array}{c}\text { até } 3 \text { horas } \\
(\mathrm{N}=14)\end{array}$ & $\begin{array}{c}>3 \text { horas } \\
(\mathrm{N}=11)\end{array}$ \\
\hline 57 & 240 \\
99 & 120 \\
665 & 147 \\
30 & 90 \\
180 & 720 \\
10 & 200 \\
30 & 93 \\
33 & 128 \\
70 & 739 \\
35 & 130 \\
15 & 180 \\
15 & \\
60 & \\
43 & 253,3 \\
95,8 & $(4 \mathrm{~h} 13 \mathrm{~min})$ \\
\hline Média &
\end{tabular}

Teste de Mann-Whitney $(p=0,001)$

ram nas primeiras 3 horas de manifestação da doença (Figura 1) e 43,6\% ocorreram em pacientes que deram entrada ao hospital com mais de 3 horas (Figura 1), podendo ter ocorrido mais de um sinal ou sintoma no mesmo paciente.

O intervalo de tempo entre a chegada ao hospital e a realização de TC foi em média de 1 hora e 55 minutos. Entre os pacientes que chegaram 
Tabela 2. Relação entre o intervalo de tempo desde o início dos sinais e sintomas até à chegada ao hospital e as complicações.

\begin{tabular}{|c|c|c|c|c|c|c|c|c|c|c|c|c|c|c|}
\hline \multirow[t]{2}{*}{ Complicações } & \multicolumn{2}{|c|}{$\begin{array}{c}\text { Bronco } \\
\text { aspiração }\end{array}$} & \multicolumn{2}{|c|}{ Pneumonia } & \multicolumn{2}{|c|}{$\begin{array}{l}\text { Infecção do } \\
\text { trato urinário }\end{array}$} & \multicolumn{2}{|c|}{ Hipotensão } & \multicolumn{2}{|c|}{ Hipertensão } & \multicolumn{2}{|c|}{ Hipertermia } & \multicolumn{2}{|c|}{ Arritmia } \\
\hline & $\mathrm{N}$ & $\%$ & $\mathrm{~N}$ & $\%$ & $\mathrm{~N}$ & $\%$ & $\mathrm{~N}$ & $\%$ & $\mathrm{~N}$ & $\%$ & $\mathrm{~N}$ & $\%$ & $\mathrm{~N}$ & $\%$ \\
\hline Até $60 \mathrm{~min}$ & 0 & 0,0 & 0 & 0,0 & 1 & 33,3 & 0 & 0,0 & 5 & 29,4 & 0 & 0.0 & 2 & 33,3 \\
\hline 1 a $2 \mathrm{~h}$. & 2 & 100 & 2 & 33,0 & 1 & 33,3 & 1 & 25,0 & 4 & 23,5 & 2 & 66,7 & 1 & 16,7 \\
\hline 2 a $3 \mathrm{~h}$. & 0 & 0,0 & 0 & 0,0 & 0 & 0,0 & 1 & 25,0 & 2 & 11,8 & 0 & 0,0 & 2 & 33,3 \\
\hline 3 a $6 \mathrm{~h}$. & 0 & 0,0 & 1 & 16,7 & 0 & 0,0 & 1 & 25,0 & 0 & 0,0 & 0 & 0,0 & 0 & 0,0 \\
\hline 6 a $12 \mathrm{~h}$. & 0 & 0,0 & 2 & 33,3 & 1 & 33,3 & 1 & 25,0 & 3 & 17,6 & 1 & 33,3 & 1 & 16,7 \\
\hline 12 a $24 \mathrm{~h}$. & 0 & 0,0 & 0 & 0,0 & 0 & 0,0 & 0 & 0,0 & 0 & 0,0 & 0 & 0,0 & 0 & 0,0 \\
\hline$>24 \mathrm{~h}$. & 0 & 0,0 & 1 & 16,7 & 0 & 0,0 & 0 & 0,0 & 2 & 11,8 & 0 & 0,0 & 0 & 0,0 \\
\hline Não informado & 0 & 0,0 & 0 & 0,0 & 0 & 0,0 & 0 & 0,0 & 1 & 5,9 & 0 & 0,0 & 0 & 0,0 \\
\hline Total & 2 & 100 & 6 & 100 & 3 & 100 & 4 & 100 & 17 & 100 & 3 & 100 & 6 & 100 \\
\hline \multirow[t]{2}{*}{ Complicações } & \multicolumn{2}{|c|}{$\begin{array}{l}\text { Hipo- } \\
\text { glicemia }\end{array}$} & \multicolumn{2}{|c|}{$\begin{array}{l}\text { Hiper- } \\
\text { glicemia }\end{array}$} & \multicolumn{4}{|c|}{$\begin{array}{c}\text { Transformação } \\
\text { hemorrágica }\end{array}$} & \multicolumn{2}{|c|}{ Outros } & \multicolumn{2}{|c|}{ Nenhuma } & & \\
\hline & $\mathrm{N}$ & $\%$ & $\mathrm{~N}$ & $\%$ & $\mathrm{~N}$ & $\%$ & $\mathrm{~N}$ & $\%$ & $\mathrm{~N}$ & $\%$ & $\mathrm{~N}$ & $\%$ & & \\
\hline Até $60 \mathrm{mim}$ & 1 & 33,3 & 2 & 22,2 & 2 & 20,0 & 1 & 20,0 & 1 & 10,0 & 1 & 9,1 & & \\
\hline 1 a $2 \mathrm{~h}$. & 1 & 33,3 & 3 & 33,3 & 3 & 30,0 & 2 & 40,0 & 3 & 30,0 & 1 & 9,1 & & \\
\hline 2 a $3 \mathrm{~h}$. & 0 & 0,0 & 2 & 22,2 & 1 & 10,0 & 1 & 20,0 & 2 & 20,0 & 0 & 0,0 & & \\
\hline 3 a $6 \mathrm{~h}$. & 0 & 0,0 & 0 & 0,0 & 1 & 10,0 & 1 & 20,0 & 0 & 0,0 & 2 & 18,2 & & \\
\hline 6 a $12 \mathrm{~h}$. & 1 & 33,3 & 2 & 22,2 & 3 & 30,0 & 0 & 0,0 & 3 & 30,0 & 4 & 36,4 & & \\
\hline 12 a 24 h. & 0 & 0,0 & 0 & 0,0 & 0 & 0,0 & 0 & 0,0 & 0 & 0,0 & 2 & 18.2 & & \\
\hline$<24 \mathrm{~h}$. & 0 & 0,0 & 0 & 0,0 & 0 & 0,0 & 0 & 0,0 & 1 & 10,0 & 1 & 9,1 & & \\
\hline Não informado & 0 & 0,0 & 1 & 11,1 & 0 & 0,0 & 0 & 0,0 & 0 & 0,0 & 0 & 0,0 & & \\
\hline Total & 3 & 100 & 9 & 100 & 10 & 100 & 5 & 100 & 10 & 100 & 11 & 100 & & \\
\hline
\end{tabular}

RNC, rebaixamento do nível de consciênciência.

no período até 3 horas desde o início dos sintomas, a realização da TC ocorreu em média 1 hora e 36 minutos após sua chegada, mostrando diferença significante $(p=0,0001)$ em relação aos pacientes que compareceram com mais de 3 horas de início dos sintomas (Tabela 1). Em 13 casos $(34,2 \%)$ não foi possível obter esta informação.

Entre as dificuldades encontradas pela população estudada até a chegada ao hospital, 6 pacientes $(15,8 \%)$ tentaram atendimento sem sucesso em outro serviço, $6(15,8 \%)$ alegaram falta de transporte, $5(13,2 \%)$ referiram que havia congestionamento de trânsito, $4(10,5 \%)$ não sabiam a gravidade, $3(7,9 \%)$ referiram que moravam ou estavam sozinhos, $6(15,8 \%)$ negaram dificuldades, $6(15,8 \%)$ apresentaram outros tipos de proble- mas, tais como: carregar a vítima ao veículo, morar longe e a demora da ambulância. Não foi possível obter esta informação de 7 pacientes $(18,4 \%)$.

As principais complicações foram infecciosas pulmonares e do trato urinário (Tabela 2). Na categoria outras complicações foram encontradas: hipocalemia, hipercalemia, aumento da pressão intracraniana, insuficiência renal aguda, cetoacidose diabética, obstipação, convulsão, insuficiência respiratória aguda, dissecção arterial extracerebral e edema agudo de pulmão.

Dos 44 procedimentos invasivos mais frequentemente realizados, $17(38,6 \%)$ corresponderam à sondagem gástrica ou enteral, $13(29,5 \%)$ necessitaram de sondagem vesical de demora, $9(20,5 \%)$ necessitaram de entubação endotraqueal, $5(11,4 \%)$ 
Tabela 3. Relação entre o intervalo de tempo desde o início dos sintomas à chegada ao hospital e o tempo de internação.

\begin{tabular}{|c|c|c|c|c|c|c|c|c|c|c|c|c|}
\hline \multirow{2}{*}{$\begin{array}{l}\text { Tempo de } \\
\text { internação }\end{array}$} & \multicolumn{2}{|c|}{ Até 3 dias } & \multicolumn{2}{|c|}{ 4-6 dias } & \multicolumn{2}{|c|}{ 7-9 dias } & \multicolumn{2}{|c|}{ 10-14 dias } & \multicolumn{2}{|c|}{15 dias } & \multicolumn{2}{|c|}{ Total } \\
\hline & $\mathrm{N}$ & $\%$ & $\mathrm{~N}$ & $\%$ & $\mathrm{~N}$ & $\%$ & $\mathrm{~N}$ & $\%$ & $\mathrm{~N}$ & $\%$ & $\mathrm{~N}$ & $\%$ \\
\hline Até $60 \mathrm{~min}$ & 2 & 28,6 & 2 & 28,36 & 0 & 0,0 & 1 & 14,3 & 3 & 42,9 & 7 & 100 \\
\hline 1 a 2 h. & 4 & 50,0 & 0 & 0,0 & 0 & 0,0 & 0 & 0,0 & 4 & 50,0 & 8 & 100 \\
\hline 2 a $3 \mathrm{~h}$. & 1 & 33,3 & 1 & 33,3 & 1 & 33,3 & 0 & 0,0 & 0 & 0,0 & 3 & 100 \\
\hline 3 a $6 \mathrm{~h}$. & 1 & 25,0 & 1 & 25,0 & 1 & 25,0 & 1 & 25,0 & 0 & 0,0 & 4 & 100 \\
\hline 6 a $12 \mathrm{~h}$. & 1 & 11,1 & 3 & 33,3 & 1 & 11,1 & 2 & 22,2 & 2 & 22,2 & 9 & 100 \\
\hline 12 a 24 h. & 0 & 0,0 & 0 & 0,0 & 0 & 0,0 & 1 & 50,0 & 1 & 50,0 & 2 & 100 \\
\hline$>24 \mathrm{~h}$. & 1 & 25,0 & 0 & 0,0 & 0 & 0,0 & 3 & 75,0 & 0 & 0,0 & 4 & 100 \\
\hline Não informado & 0 & 0,0 & 1 & 100 & 0 & 0,0 & 0 & 0,0 & 0 & 0,0 & 1 & 100 \\
\hline Total & 10 & 26,3 & 8 & 21,0 & 3 & 7,9 & 8 & 21,0 & 10 & 26,3 & 38 & 100 \\
\hline
\end{tabular}

Tabela 4. Relação entre o intervalo de tempo desde o início dos sintomas à chegada ao hospital e o destino do paciente.

\begin{tabular}{lcccccccc}
\hline Destino & \multicolumn{2}{c}{ Alta } & \multicolumn{2}{c}{ Óbito } & \multicolumn{2}{c}{ Outros } & \multicolumn{2}{c}{ Total } \\
& $\mathrm{N}$ & $\%$ & $\mathrm{~N}$ & $\%$ & $\mathrm{~N}$ & $\%$ & $\mathrm{~N}$ & $\%$ \\
\hline Até 60 min & 6 & 85,7 & 1 & 14,3 & 0 & 0,0 & 7 & 100 \\
1 a 2 h. & 6 & 75,0 & 2 & 25,0 & 0 & 0,0 & 8 & 100 \\
2 a 3 h & 2 & 66,7 & 1 & 33,3 & 0 & 0,0 & 3 & 100 \\
3 a 6 h & 3 & 75,0 & 1 & 25,0 & 0 & 0,0 & 4 & 100 \\
6 a 12 h & 6 & 66,7 & 2 & 22,2 & 1 & 11,1 & 9 & 100 \\
12 a 24 h & 2 & 100 & 0 & 0,0 & 0 & 0,0 & 2 & 100 \\
$>$ 24 h. & 2 & 50,0 & 0 & 0,0 & 2 & 50,0 & 4 & 100 \\
Não informado & 1 & 100 & 0 & 0,0 & 0 & 0,0 & 1 & 100 \\
Total & 28 & 73,7 & 7 & 18,4 & 3 & 7,9 & 38 & 100 \\
\hline
\end{tabular}

apresentavam acesso venoso central e 19 pacientes $(50 \%)$ não foram submetidos a procedimentos invasivos.

O tempo de internação foi em média de 8,5 dias (Tabela 3) e não se relacionou com o tempo de chegada ao pronto socorro desde o início dos sinais e sintomas (Tabela 2).

Dos 38 pacientes, 28 (73,7\%) tiveram alta hospitalar, $7(18,4 \%)$ morreram, 2 foram transferidos $(5,3 \%)$ e 1 paciente evadiu-se do serviço $(2,6 \%)$; a evolução também não se relacionou com o tempo de chegada ao pronto socorro desde o início dos sinais e sintomas (Tabela 4).

\section{DISCUSSÃO}

Alguns estudos demonstraram que a principal causa que restringe o uso de trombolíticos no pa- ciente com $\mathrm{AVCl}$ é o tempo de apresentação ao serviço de emergência desde o início dos sintomas, quando excede 3 horas $^{12,13}$. Diante desta janela de tempo o Food and Drug Administration (FDA) aprovou em junho de 1996 o uso do rt-PA, baseado nos resultados do National Institute of Neurologic Disorders (NINDS), que analisou os passos sequenciais a serem seguidos para um atendimento rápido e sistematizado, obedecendo à janela terapêutica, entre eles: o reconhecimento dos sinais e sintomas e ação pelo paciente ou conhecido, acesso ao cuidado, transporte ao hospital com TC, diagnóstico rápido no departamento de emergência, interpretação da TC, critérios de inclusão e tratamento ${ }^{8}$. De modo análogo ao preconizado para o atendimento de emergência cardiológica, a American Heart Associa- 
tion (AHA) também recomenda uma forma de abordagem rápida ao AVC, constituída pelos seguintes passos: detecção do início dos sinais e sintomas do evento vascular, encaminhamente através da ativação do sistema de ambulância, alerta do departamento (serviço) de emergência, coleta de dados (avaliação) e decisão sobre possíveis terapêuticas ${ }^{1}$.

Em estudo prévio no nosso serviço (19981999) ${ }^{10}$, observou-se que a média de tempo para chegada do paciente ao serviço de emergência era de 18 horas e 46 minutos e a média para a realização da TC de 5 horas e 20 minutos. Comparativamente, houve redução aproximada de 8 e 3 horas, respectivamente, mostrando que a população tem chegado mais precocemente ao hospital e, principalmente sugerindo que os médicos clínicos, neurologistas, enfermeiros, radiologistas e outros profissionais envolvidos no atendimento, têm reconhecido o evento como emergência médica. Segundo a literatura, diversos são os fatores que contribuem para a apresentação precoce do paciente ao serviço de emergência: a presença de fatores de risco, tipos de sinais e sintomas, meio de transporte utilizado, nível de escolaridade, local do acontecimento e a circunstância de indivíduos que vivem sozinhos ${ }^{7,12-14}$. Em nosso estudo, como esperado, verificamos que o principal fator de risco é a hipertensão arterial, seguida por doenças cardíacas, AVC prévio, tabagismo e diabetes mellitus, distribuídos sem diferenças quanto ao tempo. Observamos ainda que os pacientes que dão entrada ao hospital até 3 horas apresentam sinais e sintomas mais evidentes. Nenhum paciente que chegou ao hospital com mais de 12 horas desde o início dos sintomas morreu e a maioria que ficou internada por mais de 15 dias chegou precocemente, sugerindo que pacientes mais graves chegam antes.

Diferentemente de outros países, onde o reconhecimento do AVC é realizado através da aplicação da escala de Cincinnati por paramédicos no âmbito pré-hospitalar, agilizando o transporte e o cuidado a este paciente ${ }^{15-17}$, em nosso meio o acesso à ambulância é restrito e a maioria utiliza-se do transporte privado. Verificamos também que as principais dificuldades enfrentadas pela população atendida neste hospital são a falta de transporte, a procura de outros serviços onde não obtiveram terapêutica adequada e o congestionamento de trânsito. Poucos são os estudos realizados sobre a hospitalização e complica- ções precoces do AVC isquêmico. Percebemos que há uniformidade em relação às complicações apresentadas pelos pacientes, independentemente do tempo de chegada. Sabemos que é de fundamental importância a realização do controle dos sinais vitais e o controle rápido das alterações funcionais. Estudos mostram que a hipertensão deve ser controlada, mantendo-se assim; a hipertermia, a hipoglicemia e a hiperglicemia pioram o grau de lesão neurológica pela ativação de reações imunomediadas, diminuição de substrato energético, aumento do edema citotóxico e vasogênico, aumento na produção de radicais livres ${ }^{18-20}$. $A$ equipe de enfermagem tem papel preponderante no atendimento do paciente com AVC, participando ativamente do controle rigoroso dos sinais vitais, cuidando para que a realização de procedimentos invasivos seja criteriosa, para diminuir os focos infecciosos. Verificamos em nosso estudo que metade dos pacientes necessitaram de procedimentos invasivos. Para melhorar a qualidade de assistência e evitar as infecções, faz-se necessária a orientação, em forma de protocolos, quanto à realização destes procedimentos e seus cuidados. Portanto, alerta-se não apenas para o tratamento medicamentoso, mas também para o controle de todos os fatores que podem agravar a lesão neurológica. Salientamos o esforço conjunto da equipe de atendimento ao paciente no setor de emergência e a motivação para se empreender um trabalho árduo, com menor risco de complicação possível.

Verificou-se que o tempo médio de internação para pacientes com AVC isquêmico foi relativamente grande no nosso serviço, possivelmente devido à viéses de seleção de uma população atendida em hospital terciário. Os dados atuais, de maneira geral, quando comparados aos de 1999, sugerem mudança de mentalidade no atendimento ao paciente com AVCI no HSP. Este trabaIho demonstrou que, em nosso serviço, o $\mathrm{AVCl}$ já é considerado emergência médica e que cerca da metade dos pacientes têm chegado ao hospital em tempo menor ou igual a 3 horas desde o início dos sintomas. Quando o paciente chega ao hospital dentro da janela terapêutica, a TC para estes pacientes tem sido realizada em tempo hábil para o tratamento trombolítico. Demonstrouse também que as complicações podem aumentar o tempo de hospitalização, sendo de grande importância que elas sejam controladas e evitadas através da implantação e principalmente di- 


\section{vulgação entre todos os membros da equipe de protocolos de atendimento ao paciente com AVC no setor de emergência.}

\section{REFERÊNCIAS}

1. American Heart Association. 2000 Heart and Stroke Statistical Update. Dallas: American Heart Association, 1999.

2. Mennot A, Jacobs Jr DR, Blackburn H, Kromheit D, Nissinen A, Medelgovic S. Twenty-five year prediction of stroke death in the seven country study: the role of blood pressure and its changes: Stroke 1996;27:381-387.

3. Lessa, I. Epidemiologia das doenças cerebrovasculares no Brasil. Rev Soc Cardiol Est S Paulo 1999;9:509-518.

4. Matchar DB, Duncan PW. Cost of stroke. Stroke Clinical Updates, 1994;5:9-12.

5. Yatsu FM. Treatment and prevention of stroke. In Rowland LP (ed). Merrit's textbook of neurology. 9 Ed. Baltimore: Williams \& Wilkins, 1995:272-275

6. Gelmers HJ, Gorter K, Weerdt CJ, Wiezer JHA. A controlled trial of nimodipine in acute ischemic stroke. N Engl J Med 1998;318:208-217.

7. Feldmann E, Gorson N, Brooks J, Brass L, Fayad PB, Sawaya KL. Factors associated with early presentation of acute stroke. Stroke 1993; 24:1805-1810.

8. Kohtari RU, Hacke W, Brott T et al. Stroke. Ann Emerg Med 2001; 37:S137-S144.

9. Ferro JM. Conduta inicial no paciente com acidente vascular cerebral isquêmico agudo. Rev Soc Cardiol Est S Paulo 1999;9:570-577.

10. Leopoldino JSF, Fukujima MM, Silva GS, Prado GF. Time of pre- sentation of stroke patients in São Paulo Hospital. Arq Neuropsiquiatr 2003;61:186-187.

11. Sociedade Brasileira de Doenças Cerebrovasculares. Primeiro consenso brasileiro do tratamento da fase aguda do acidente vascular cerebral. Arq Neuropsiquiatr 2001;59:972-980.

12. Harper GD, Haigh RA, Potter DM, Castleden CM. Factors delaying hospital admission after stroke in Leicestershire. Stroke 1992;23:835838 .

13. O'Connor RE, McGraw P, Edelsohn L. Trombolytic therapy for acute ischemic stroke: why the majority of patients remain ineligible for treatment. Ann Emerg Med 1999;33:9-14.

14. Alberts, MJ, Bertels C, Dawson DV. An analysis of time of presentation after stroke. JAMA. 1990;263:65-68.

15. Kothari R, Jauch E, Broderick J et al. Acute stroke: delays to presentation and emergency department evaluation. Ann Emerg Med 1999; 33:3-8

16. Kothari RU, Pancioli A, Liu T, Brott T, Broderick J. Cincinnati prehospital stroke scale: reproducibility and validity. Ann Emerg Med 1999 33:373-378

17. Kothari R, Barsan W, Brott T, Broderick J, Ashbrock S. Frequency and accuracy of prehospital diagnosis of acute stroke. Stroke 1995;26:937-941.

18. Coimbra C, Drake M, Boris Möller F, Wieloch T. Long-lasting neuroprotective effectof postischemic hypothermia and treatment with na anti-inflamatory/antipyretic drug: evidence for chronic encephalopathic processes following cerebral ischemia. Stroke 1996;27:1578-1585.

19. Ginsberg MD, Busto R. Combating hyperthermia in acute stroke Stroke 1998;29:529-534

20. Cherian L, Goodman JC, Robertson CS. Hyperglycemia increases brain injury caused by secondary ischemia after cortical impact injury in rats. Crit Care Med 1997;25:1378-1383. 